\title{
Differentiation of hepatocellular adenoma and focal nodular hyperplasia using ${ }^{18}$ F-fluorocholine PET/CT
}

\author{
Jacomina W. van den Esschert • Matthanja Bieze • \\ Ulrich H. Beuers • Thomas M. van Gulik • \\ Roelof J. Bennink
}

Received: 7 June 2010 / Accepted: 26 July 2010 / Published online: 18 August 2010

(C) The Author(s) 2010. This article is published with open access at Springerlink.com

\begin{abstract}
The aim of this pilot study was to evaluate the use of PET/CT with ${ }^{18} \mathrm{~F}$-fluorocholine in the differentiation of hepatocellular adenoma (HCA) from focal nodular hyperplasia (FNH). Patients with liver lesions larger than $2 \mathrm{~cm}$ suspicious for HCA or FNH were prospectively included. All patients underwent PET/CT with ${ }^{18} \mathrm{~F}$-fluorocholine and histopathological diagnosis was obtained by either liver biopsy or surgery. The ratios between the maximum standardized uptake value (SUV) of the lesion and the mean SUV of normal liver parenchyma were calculated and a receiver operating characteristic (ROC) curve analysis was performed. Ten patients with FNH and 11 with HCA were included. The mean SUV ratio was $1.68 \pm 0.29( \pm \mathrm{SD})$ for FNH and $0.88 \pm 0.18$ for HCA $(p<0.001)$. An SUV ratio cut-
\end{abstract}

J. W. van den Esschert $\cdot$ M. Bieze $\cdot$ T. M. van Gulik $(\square)$

Department of Surgery, Academic Medical Center Amsterdam,

Meibergdreef 9,

1105AZ Amsterdam, The Netherlands

e-mail: t.m.vangulik@amc.uva.nl

J. W. van den Esschert

e-mail: j.w.vandenesschert@amc.uva.nl

M. Bieze

e-mail: m.bieze@amc.uva.nl

U. H. Beuers

Department of Hepatology,

Academic Medical Center Amsterdam,

Meibergdreef 9,

1105AZ Amsterdam, The Netherlands

e-mail: u.h.beuers@amc.uva.nl

R. J. Bennink

Department of Nuclear Medicine,

Academic Medical Center Amsterdam,

Meibergdreef 9,

1105AZ Amsterdam, The Netherlands

e-mail: r.bennink@amc.uva.nl off value between 1.12 and 1.22 differentiated patients with FNH from those with HCA with $100 \%$ sensitivity and $100 \%$ specificity. This pilot study showed that PET/CT with ${ }^{18} \mathrm{~F}$ fluorocholine can differentiate HCA from FNH.

Keywords Hepatology · Benign liver neoplasms .

Liver cell adenoma $\cdot$ Nuclear medicine
Abbreviations
PET Positron emission tomography
CT Computed tomography
FNH Focal nodular hyperplasia
HCA Hepatocellular adenoma
SUV Standardized uptake value
ROC Receiver operating characteristics

\section{Introduction}

Hepatocellular adenoma (HCA) and focal nodular hyperplasia (FNH) are benign hypervascular liver lesions that predominantly occur in young and middle-aged women. Differentiation of these two tumours using radiological imaging modalities may be difficult because of radiological features shared by both tumours [1]. The frequency with which the diagnosis is in question after routine radiological examination is unclear. Recently it has been reported that MRI with a hepatospecific contrast agent distinguishes HCA from FNH with a sensitivity and specificity of $96.9 \%$ and $100 \%$, respectively. However, the diagnosis of most of these lesions was based solely on imaging results without histological confirmation [2]. Differentiation is crucial to decide on appropriate management because of the different therapeutic consequences. FNH is a strictly benign liver lesion and complications are very rare. Conservative 
treatment is therefore justified, in the absence of mechanical symptoms. On the other hand, HCA carries a risk of spontaneous bleeding [3] and malignant transformation, especially when the lesion is larger than $5 \mathrm{~cm}$ [4]. Therefore, resection of a HCA larger than $5 \mathrm{~cm}$ is usually advocated.

When radiological analysis remains inconclusive, a liver biopsy is required to establish the diagnosis. This is an invasive procedure with associated risks. Therefore, there is a need for accurate noninvasive diagnostic imaging techniques. In previous studies, the use of the ${ }^{99} \mathrm{Tc}$ sulphur-colloid scan was found not to be helpful in diagnosis because many lesions showed atypical uptake of the tracer [5]. In our experience, the use of ${ }^{99} \mathrm{Tc}$ mebrofenin is not accurate enough, probably due to inferior resolution of SPECT or biliary excretion on hepatobiliary scintigraphy masking smaller centrally located lesions. Bumsel and et al. recently suggested that $\mathrm{PET} / \mathrm{CT}$ using the tracer ${ }^{18} \mathrm{~F}$ fluoromethylcholine $\left({ }^{18} \mathrm{~F}-\mathrm{FCH}\right)$ may be able to differentiate between FNH and HCA [6]. In this pilot study, we prospectively assessed the use of PET/CT with ${ }^{18} \mathrm{~F}-\mathrm{FCH}$ with semiquantitative uptake measurements in patients in whom the diagnosis FNH or HCA was considered.

\section{Materials and methods}

Patients

This pilot study was part of a prospective, single-centre trial which aimed to develop a diagnostic algorithm for patients with suspicion of FNH or HCA. The study protocol was approved by the medical ethics committee of the Academic Medical Center Amsterdam. Patients over 18 years of age with suspicion of FNH or HCA larger than $2 \mathrm{~cm}$ in diameter based on radiological imaging modalities were included in the study after written informed consent had been obtained. When suspected of malignancy, based on clinical history, imaging studies or elevated plasma alpha-fetoprotein or CEA levels, the patient was excluded.

\section{PET/CT procedure}

An injection solution of 2,000-3,000 $\mathrm{MBq}$ of $\left[{ }^{18} \mathrm{~F}\right]-$ fluorocholine with a radiochemical purity of $98 \%$ or more was synthesized. PET/CT was performed using a Philips Gemini TF-16 PET/CT scanner (Philips Medical Systems, Eindhoven, The Netherlands) with a spatial resolution near

Table 1 Patient characteristics and PET/CT data

\begin{tabular}{|c|c|c|c|c|c|c|c|c|c|}
\hline Patient & Gender & $\begin{array}{l}\text { Age } \\
\text { (years) }\end{array}$ & $\begin{array}{l}\text { Tumour location } \\
\text { (segment) }\end{array}$ & $\begin{array}{l}\text { Tumour } \\
\text { size }(\mathrm{cm})\end{array}$ & $\begin{array}{l}\mathrm{SUV}_{\text {mean }} \\
\text { liver }\end{array}$ & $\begin{array}{l}\mathrm{SUV}_{\max } \\
\text { tumour }\end{array}$ & $\begin{array}{l}\text { SUV } \\
\text { ratio }\end{array}$ & $\begin{array}{l}\text { Histopathological } \\
\text { diagnosis }\end{array}$ & Treatment \\
\hline 1 & $\mathrm{~F}$ & 29 & 2 & $5.3 \times 4.2$ & 10.61 & 6.03 & 0.57 & $\mathrm{HCA}$ & Segmental resection \\
\hline 2 & $\mathrm{~F}$ & 45 & 7 & $5.0 \times 6.0$ & 8.77 & 5.49 & 0.63 & $\mathrm{HCA}$ & Segmental resection \\
\hline 3 & $\mathrm{~F}$ & 33 & 8 & $5.5 \times 4.5$ & 12.87 & 8.66 & 0.67 & $\mathrm{HCA}$ & Right hemihepatectomy \\
\hline 4 & $\mathrm{~F}$ & 35 & 2 & $6.0 \times 7.5$ & 8.86 & 7.57 & 0.85 & $\mathrm{HCA}$ & Segmental resection \\
\hline 5 & $\mathrm{~F}$ & 49 & 8 & $7.0 \times 5.9$ & 10.56 & 9.24 & 0.88 & $\mathrm{HCA}$ & Right hemihepatectomy \\
\hline 6 & $\mathrm{~F}$ & 40 & 3 & $2.3 \times 1.9$ & 12.47 & 11.52 & 0.92 & $\mathrm{HCA}$ & Observation \\
\hline 7 & $\mathrm{~F}$ & 41 & 7 & $2.3 \times 1.4$ & 8.45 & 7.93 & 0.94 & $\mathrm{HCA}$ & Observation \\
\hline 8 & $\mathrm{~F}$ & 51 & 8 & $8.5 \times 6.5$ & 10.95 & 10.55 & 0.96 & $\mathrm{HCA}$ & Right hemihepatectomy \\
\hline 9 & $\mathrm{~F}$ & 45 & 3 & $6.0 \times 7.0$ & 9.36 & 9.27 & 0.99 & $\mathrm{HCA}$ & Observation \\
\hline 10 & $\mathrm{~F}$ & 41 & 6 & $8.0 \times 4.0$ & 11.91 & 12.96 & 1.09 & $\mathrm{HCA}$ & Segmental resection \\
\hline 11 & $\mathrm{~F}$ & 40 & 1 & $11.4 \times 9.3$ & 11.48 & 12.90 & 1.12 & $\mathrm{HCA}$ & Enucleation of the tumour \\
\hline 12 & $\mathrm{~F}$ & 27 & 4 & $4.2 \times 4.5$ & 16.09 & 19.62 & 1.22 & $\mathrm{FNH}$ & Observation \\
\hline 13 & F & 38 & 1 & $5.7 \times 6.8$ & 7.21 & 10.16 & 1.41 & FNH & Enucleation of the tumour \\
\hline 14 & $\mathrm{~F}$ & 38 & 4 & $2.4 \times 2.2$ & 7.08 & 10.29 & 1.45 & FNH & Enucleation of the tumour \\
\hline 15 & $\mathrm{~F}$ & 22 & 8 & $6.2 \times 5.0$ & 6.70 & 10.51 & 1.57 & FNH & Right hemihepatectomy \\
\hline 16 & $\mathrm{~F}$ & 41 & 5 & $7.8 \times 7.2$ & 10.68 & 17.25 & 1.62 & FNH & Observation \\
\hline 17 & $\mathrm{~F}$ & 52 & 7 & $6.4 \times 6.3$ & 8.89 & 15.15 & 1.70 & FNH & Observation \\
\hline 18 & $\mathrm{~F}$ & 44 & 5 & $3.1 \times 2.7$ & 10.00 & 18.38 & 1.84 & FNH & Observation \\
\hline 19 & $\mathrm{~F}$ & 45 & 8 & $7.1 \times 7.4$ & 6.82 & 12.76 & 1.87 & FNH & Observation \\
\hline 20 & $\mathrm{~F}$ & 40 & 2 & $3.7 \times 3.1$ & 8.43 & 16.29 & 1.93 & FNH & Enucleation of the tumour \\
\hline 21 & $\mathrm{~F}$ & 36 & $7 / 8$ & $10.5 \times 6.3$ & 9.24 & 20.39 & 2.21 & FNH & Observation \\
\hline
\end{tabular}


Fig. $1 \mathrm{CT}$ and PET/CT images of a patient with FNH (a) and HCA (b) 15 min after i.v. injection of $150 \mathrm{MBq}{ }^{18} \mathrm{~F}-\mathrm{FCH}$. The FNH clearly shows increased uptake of the choline tracer. The HCA is hardly distinguishable from, but relatively cold compared to normal liver tissue
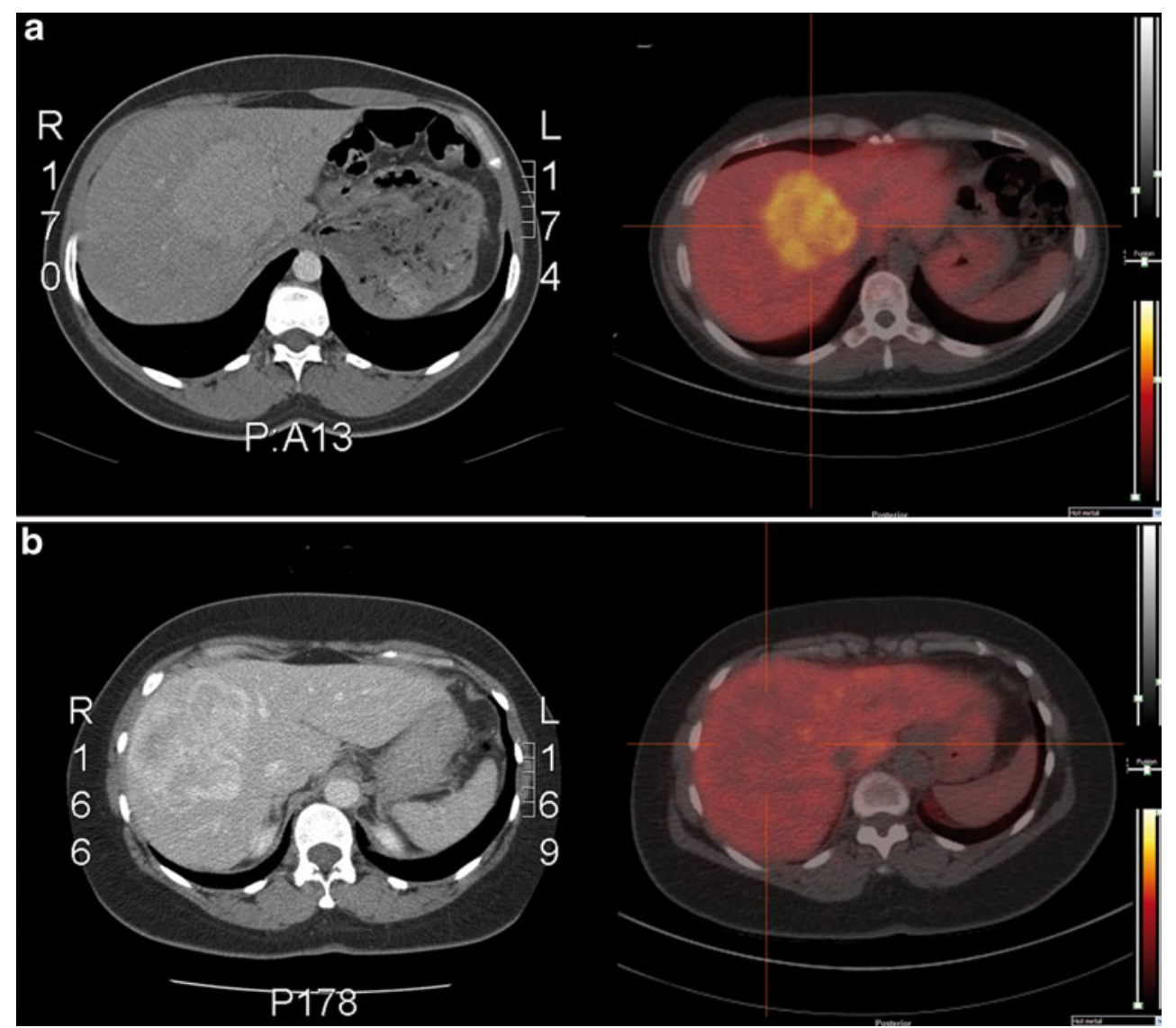

the centre of the field of view of $4.8 \mathrm{~mm}$ in transverse and axial directions. A CT transmission scan in the supine position was acquired from the mid-thorax to the midabdomen, encompassing the entire liver. The 12-channel helical CT scanning parameters were: $120 \mathrm{kVp}, 50 \mathrm{~mA} /$ slice, rotation time $0.75 \mathrm{~s}$, slice thickness/interval $5.0 \mathrm{~mm}$. No intravenous contrast agent was used for the CT. At $15 \mathrm{~min}$ after intravenous injection of $150 \mathrm{MBq}$ of ${ }^{18} \mathrm{~F}$ $\mathrm{FCH}$, emission scans were acquired from the mid-thorax to the mid-abdomen, encompassing the entire liver over three or four bed positions at $3 \mathrm{~min}$ per position. Images were reconstructed using a list-mode version of a maximum likelihood expectation maximization algorithm with a time-of-flight kernel applied in both the forward and back-projection operations. CT data were used for attenuation correction.

\section{PET/CT evaluation}

Images were analysed in consensus blinded for outcome on a Hermes workstation using Hybrid viewer software (Hermes Medical Solutions, Stockholm, Sweden). The standardized uptake value (SUV) was determined in the liver lesions and in surrounding nonaffected liver tissue volumes. Choline is normally continuously metabolized in the liver. The amount of choline used depends on the metabolic need, and therefore is not necessarily equal between patients. To allow for this different baseline choline consumption, we used the SUV ratio in our analysis. The SUV ratio was calculated by dividing the maximum SUV of the lesion $\left(\mathrm{SUV}_{\max }\right.$ tumour) by the mean SUV of the normal surrounding liver tissue (SUV $\mathrm{Sean}_{\text {liver). }}$

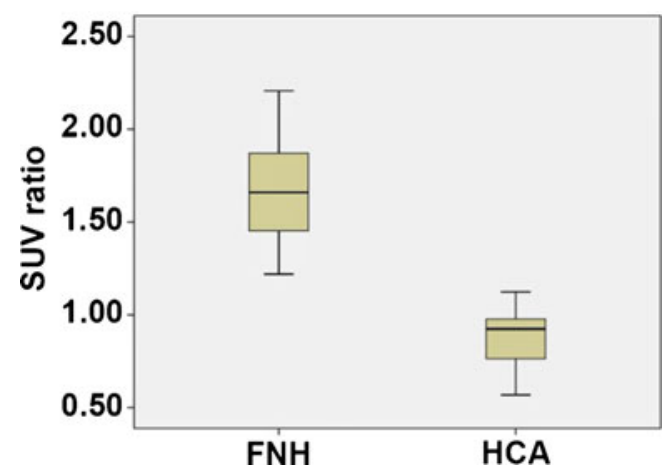

Fig. 2 Boxplot differentiating FNH and HCA in terms of choline metabolic activity of the tumour. The cut-off value of the SUV ratio with $100 \%$ sensitivity and $100 \%$ specificity lies between 1.12 to 1.22 


\section{Gold standard}

The gold standard for the diagnosis of FNH and HCA is histopathological evaluation. A histological specimen was obtained by liver biopsy or liver surgery. If multiple lesions were present in the liver, only those with a histopathologically confirmed diagnosis were scored using the PET/CT scan. The outcome of the PET/CT scan was compared with the gold standard.

\section{Statistical analysis}

Statistical analysis was performed with Statistical Package for Social Sciences (SPSS, version 16.0.2.1). Continuous data were tested for normality of distribution and equality of variances using Levene's test, and compared using an independent sample $t$-test, and are expressed as means $\pm \mathrm{SD}$. All statistical tests were two-tailed, and were evaluated at the $5 \%$ level of significance. A receiver operating characteristic (ROC) curve analysis was performed.

\section{Results}

\section{Patient characteristics}

Included in the study were 21 patients in the period between May 2009 and January 2010. All patients were female and their mean age was 39 years (range 2252 years). Patient characteristics are shown in Table 1.

\section{PET/CT performance}

Ten patients had histologically proven FNH and 11 had HCA. There were no complications after the liver biopsies. The mean SUV ratios were $1.68 \pm 0.29$ (range 1.22-2.21) for FNH and $0.88 \pm 0.18$ (range $0.57-1.12$ ) for HCA $(p<0.001)$. All FNH therefore showed increased uptake and most HCAs showed similar or decreased uptake of the choline tracer in comparison to the surrounding liver tissue (Fig. 1). ROC curve analysis suggested that a SUV ratio cut-off value between 1.12 and 1.22 differentiated patients with FNH from those with HCA with $100 \%$ sensitivity and $100 \%$ specificity. The SUV ratios for each group are shown in Fig. 2.

\section{Discussion}

This study shows that PET/CT with ${ }^{18} \mathrm{~F}-\mathrm{FCH}$ can differentiate FNH from HCA. All HCAs showed an SUV ratio $\leq 1.12$ and all FNH showed an SUV ratio $\geq 1.22$. The $100 \%$ sensitivity and specificity based on visual interpretation are in accordance with the findings of Bumsel et al. [6]. They found an intense and early uptake of FCH by all FNH and no uptake by adenoma or telangiectatic FNH. The latter lesion has, however, recently been reclassified as HCA [7]. The results of both studies are promising, but must be considered carefully because of the small sample size. In our ongoing study we will further assess the accuracy of $\mathrm{PET} / \mathrm{CT}$ with ${ }^{18} \mathrm{~F}-\mathrm{FCH}$ in more patients.

The question arises as to the phenomenon that underlies the difference in choline uptake. Choline is used in the synthesis of phosphatidylcholine and sphingomyelin, two phospholipids that are structural components of all human cell membranes. Malignant tumour cells are known to show rapid cell duplication and therefore have a higher uptake of choline as a substrate for the cell membranes. However, HCA and especially FNH are slowly proliferating liver tumours making this duplication theory unlikely. Another theory is a difference in perfusion, since choline uptake is flow-dependent [8]. On hepatobiliary scintigraphy, FNH shows increased flow, in contrast to HCA. On contrast-enhanced ultrasonography, both FNH and HCA show rapid filling of the lesion, but an HCA also shows rapid wash-out of the contrast material [9]. Finally, there could be a difference in the metabolism of very low-density lipoproteins (VLDL) in the tumours. The membrane of VLDL particles mainly consists of phospholipids. Although less plausible, it could be that FNH has an increased synthesis of VLDL particles.

A drawback of the use of PET/CT using noncontrastenhanced low-dose CT is that the tumours might be difficult to localize. In such cases image fusion with a contrastenhanced CT or MRI scan is needed to locate the lesions.

Based on the findings of this study, we can conclude that $\mathrm{PET} / \mathrm{CT}$ with ${ }^{18} \mathrm{~F}$-fluorocholine is able to differentiate HCA from FNH with high sensitivity and specificity. Study of a larger patient series is, however, needed to confirm these results.

Acknowledgment The authors wish to acknowledge the support of Dr. A.D. Windhorst and the Radionuclide Center at the Free University of Amsterdam for synthesis of $\mathrm{FCH}$.

Financial support None.

Open Access This article is distributed under the terms of the Creative Commons Attribution Noncommercial License which permits any noncommercial use, distribution, and reproduction in any medium, provided the original author(s) and source are credited.

\section{References}

1. van den Esschert JW, van Gulik TM, Phoa SS. Imaging modalities for focal nodular hyperplasia and hepatocellular adenoma. Dig Surg 2010;27:46-55. 
2. Grazioli L, Morana G, Kirchin MA, Schneider G. Accurate differentiation of focal nodular hyperplasia from hepatic adenoma at gadobenate dimeglumine-enhanced MR imaging: prospective study. Radiology 2005;236:166-77.

3. Shortell CK, Schwartz SI. Hepatic adenoma and focal nodular hyperplasia. Surg Gynecol Obstet 1991;173:426-31.

4. Foster JH, Berman MM. The malignant transformation of liver cell adenomas. Arch Surg 1994;129:712-7.

5. Herman P, Pugliese V, Machado MA, Montagnini AL, Salem MZ, Bacchella T, et al. Hepatic adenoma and focal nodular hyperplasia: differential diagnosis and treatment. World J Surg 2000;24:372-6.

6. Bumsel F, Huchet V, Arrive L, Wendum D, Paye F, Poupon R, et al. Positron emission tomography (PET/CT) using fluro-choline allows to differentiate between adenoma and focal nodular hyperplasia (abstract 437). Hepatology 2007;46(Suppl. 1):433A.

7. Bioulac-Sage P, Rebouissou S, Thomas C, Blanc JF, Saric J, Sa $\mathrm{CA}$, et al. Hepatocellular adenoma subtype classification using molecular markers and immunohistochemistry. Hepatology 2007;46:740-8.

8. Sutinen E, Nurmi M, Roivainen A, Varpula M, Tolvanen T, Lehikoinen $\mathrm{P}$, et al. Kinetics of $[(11) \mathrm{C}]$ choline uptake in prostate cancer: a PET study. Eur J Nucl Med Mol Imaging 2004;31:317-24.

9. Trillaud H, Bruel JM, Valette PJ, Vilgrain V, Schmutz G, Oyen R, et al. Characterization of focal liver lesions with SonoVueenhanced sonography: international multicenter-study in comparison to CT and MRI. World J Gastroenterol 2009;15:3748-56. 